\title{
Spectral saturation: inverting the spectral Turán theorem
}

\author{
Vladimir Nikiforov \\ Department of Mathematical Sciences \\ University of Memphis, Memphis TN, USA \\ vnikifrv@memphis.edu
}

Submitted: Nov 28, 2007; Accepted: Feb 26, 2009; Published: Mar 13, 2009

Mathematics Subject Classifications: 05C50, 05C35

\begin{abstract}
Let $\mu(G)$ be the largest eigenvalue of a graph $G$ and $T_{r}(n)$ be the $r$-partite Turán graph of order $n$.

We prove that if $G$ is a graph of order $n$ with $\mu(G)>\mu\left(T_{r}(n)\right)$, then $G$ contains various large supergraphs of the complete graph of order $r+1$, e.g., the complete $r$-partite graph with all parts of size $\log n$ with an edge added to the first part.

We also give corresponding stability results.
\end{abstract}

Keywords: complete r-partite graph; stability, spectral Turán's theorem; largest eigenvalue of a graph.

\section{Introduction}

This note is part of an ongoing project aiming to build extremal graph theory on spectral basis, see, e.g., [3], [13, 18].

Let $\mu(G)$ be the largest adjacency eigenvalue of a graph $G$ and $T_{r}(n)$ be the $r$-partite Turán graph of order $n$. The spectral Turán theorem [15] implies that if $G$ is a graph of order $n$ with $\mu(G)>\mu\left(T_{r}(n)\right)$, then $G$ contains a $K_{r+1}$, the complete graph of order $r+1$.

On the other hand, it is known (e.g., [2], [4], [9], [12]) that if $e(G)>e\left(T_{r}(n)\right)$, then $G$ contains large supergraphs of $K_{r+1}$. It turns out that essentially the same results also follow from the inequality $\mu(G)>\mu\left(T_{r}(n)\right)$.

Recall first a family of graphs, studied initially by Erdős [7] and recently in [2]: an $r$-joint of size $t$ is the union of $t$ distinct $r$-cliques sharing an edge. Write $j s_{r}(G)$ for the maximum size of an $r$-joint in a graph $G$. Erdös [7], Theorem $3^{\prime}$, showed that if $G$ is a graph of sufficiently large order $n$ satisfying $e(G)>e\left(T_{r}(n)\right)$, then $j s_{r+1}(G)>$ $n^{r-1} /(10(r+1))^{6(r+1)}$. 
Here is a explicit spectral analogue of this result.

Theorem 1 Let $r \geq 2, n>r^{15}$, and $G$ be a graph of order $n$. If $\mu(G)>\mu\left(T_{r}(n)\right)$, then $j s_{r+1}(G)>n^{r-1} / r^{2 r+4}$.

Erdös [4] introduced yet another graph related to Turán's theorem: let $K_{r}^{+}\left(s_{1}, \ldots, s_{r}\right)$ be the complete $r$-partite graph with parts of sizes $s_{1} \geq 2, s_{2}, \ldots, s_{r}$, with an edge added to the first part. The extremal results about this graph given in [4] and [9] were recently extended in [12] to:

Let $r \geq 2,2 / \ln n \leq c \leq r^{-(r+7)(r+1)}$, and $G$ be a graph of order $n$. If $G$ has $t_{r}(n)+1$ edges, then $G$ contains a $K_{r}^{+}\left(\lfloor c \ln n\rfloor, \ldots,\lfloor c \ln n\rfloor,\left\lceil n^{1-\sqrt{c}}\right\rceil\right)$.

Here we give a similar spectral extremal result.

Theorem 2 Let $r \geq 2,2 / \ln n \leq c \leq r^{-(2 r+9)(r+1)}$, and $G$ be a graph of order $n$. If $\mu(G)>\mu\left(T_{r}(n)\right)$, then $G$ contains a $K_{r}^{+}\left(\lfloor c \ln n\rfloor, \ldots,\lfloor c \ln n\rfloor,\left\lceil n^{1-\sqrt{c}}\right\rceil\right)$.

As an easy consequence of Theorem 2 we obtain

Theorem 3 Let $r \geq 2, c=r^{-(2 r+9)(r+1)}, n \geq e^{2 / c}$, and $G$ be a graph of order $n$. If $\mu(G)>\mu\left(T_{r}(n)\right)$, then $G$ contains a $K_{r}^{+}(\lfloor c \ln n\rfloor, \ldots,\lfloor c \ln n\rfloor)$.

Theorems 1, 2, and 3 have corresponding stability results.

Theorem 4 Let $r \geq 2,0<b<2^{-10} r^{-6}, n \geq r^{20}$, and $G$ be a graph of order $n$. If $\mu(G)>(1-1 / r-b) n$, then $G$ satsisfies one of the conditions:

(a) $j s_{r+1}(G)>n^{r-1} / r^{2 r+5}$;

(b) $G$ contains an induced $r$-partite subgraph $G_{0}$ of order at least $\left(1-4 b^{1 / 3}\right) n$ with minimum degree $\delta\left(G_{0}\right)>\left(1-1 / r-7 b^{1 / 3}\right) n$.

Theorem 5 Let $r \geq 2,2 / \ln n \leq c \leq r^{-(2 r+9)(r+1)} / 2,0<b<2^{-10} r^{-6}$, and $G$ be a graph of order $n$. If $\mu(G)>(1-1 / r-b) n$, then $G$ satsisfies one of the conditions:

(a) $G$ contains a $K_{r}^{+}\left(\lfloor c \ln n\rfloor, \ldots,\lfloor c \ln n\rfloor,\left\lceil n^{1-2 \sqrt{c}}\right\rceil\right)$;

(b) $G$ contains an induced $r$-partite subgraph $G_{0}$ of order at least $\left(1-4 b^{1 / 3}\right) n$ with minimum degree $\delta\left(G_{0}\right)>\left(1-1 / r-7 b^{1 / 3}\right) n$.

Theorem 6 Let $r \geq 2, c=r^{-(2 r+9)(r+1)} / 2,0<b<2^{-10} r^{-6}, n \geq e^{2 / c}$, and $G$ be a graph of order $n$. If $\mu(G)>(1-1 / r-b) n$, then one of the following conditions holds:

(a) $G$ contains a $K_{r}^{+}(\lfloor c \ln n\rfloor, \ldots,\lfloor c \ln n\rfloor)$;

(b) $G$ contains an induced $r$-partite subgraph $G_{0}$ of order at least $\left(1-4 b^{1 / 3}\right) n$ with minimum degree $\delta\left(G_{0}\right)>\left(1-1 / r-7 b^{1 / 3}\right) n$. 


\section{Remarks}

- Obviously Theorems 1,2 , and 3 are tight since $T_{r}(n)$ contains no $(r+1)$-cliques.

- Theorems 2, 3, 5, and 6 are essentially best possible since for every $\varepsilon>0$, choosing randomly a graph $G$ of order $n$ with $e(G)=\left\lceil(1-\varepsilon) n^{2} / 2\right\rceil$ edges we see that $\mu(G)>(1-\varepsilon) n$, but $G$ contains no $K_{2}(c \ln n, c \ln n)$ for some $c>0$, independent of $n$.

- In Theorem 1, it is not known what is the best possible value of $j s_{r+1}(G)$, given $G$ is a graph of order $n$ and $\mu(G)>\mu\left(T_{r}(n)\right)$.

- Theorem 1 implies in turn spectral versions of other known results, like Theorem 3.8 in [8]:

Every graph $G$ of order $n$ with $\mu(G)>\mu\left(T_{r}(n)\right)$ contains cn distinct $(r+1)$ cliques sharing an $r$-clique, where $c>0$ is independent of $n$.

- It is not difficult to show that if $G$ is a graph of order $n$, then the inequality $e(G)>$ $e\left(T_{r}(n)\right)$ implies the inequality $\mu(G)>\mu\left(T_{r}(n)\right)$. Therefore, Theorems 1-6 imply the corresponding nonspectral extremal results of [12] with narrower ranges of the parameters.

- The relations between $c$ and $n$ in Theorems 2 and 5 need some explanation. First, for fixed $c$, they show how large must be $n$ so that the vertex classes of the required $K_{r}^{+}(s, \ldots s, t)$ are nonempty. But also $c$ may depend on $n$, e.g., letting $c=1 / \ln \ln n$, the conclusion is meaningful for sufficiently large $n$.

- Note that, in Theorems 2 and 5, if the conclusion holds for some $c$, it holds also for $0<c^{\prime}<c$, provided $n$ is sufficiently large, i.e., as $n$ grows, we can find a larger and more lopsided $K_{r}^{+}(s, \ldots s, t)$;

- The stability conditions (b) in Theorems 4, 5, and 6 are stronger than the conditions in the stability theorems of [6], [19] and [11]. Indeed, in all these theorems, condition (b) implies that $G_{0}$ is an induced, almost balanced, and almost complete $r$-partite graph containing almost all the vertices of $G$;

- The exponents $1-\sqrt{c}$ and $1-2 \sqrt{c}$ in Theorems 2 and 5 are far from the best ones, but are simple.

The next section contains notation and results needed to prove the theorems. The proofs are presented in Section 3.

\section{Preliminary results}

Our notation follows [1]. Given a graph $G$, we write: 
- $V(G)$ for the vertex set of $G$ and $|G|$ for $|V(G)|$;

- $E(G)$ for the edge set of $G$ and $e(G)$ for $|E(G)|$;

- $d(u)$ for the degree of a vertex $u$;

- $\delta(G)$ for the minimum degree of $G$;

- $k_{r}(G)$ for the number of $r$-cliques of $G$;

- $K_{r}\left(s_{1}, \ldots, s_{r}\right)$ for the complete $r$-partite graph with parts of sizes $s_{1}, \ldots, s_{r}$.

The following facts play crucial roles in our proofs.

Fact 7 ([15], Theorem 1) Every graph $G$ of order $n$ with $\mu(G)>\mu\left(T_{r}(n)\right)$ contains a $K_{r+1}$.

Fact 8 ([16], Theorem 5) Let $0<\alpha \leq 1 / 4, \quad 0<\beta \leq 1 / 2, \quad 1 / 2-\alpha / 4 \leq \gamma<1$, $K \geq 0, \quad n \geq(42 K+4) / \alpha^{2} \beta$, and $G$ be a graph of order $n$. If

$$
\mu(G)>\gamma n-K / n \quad \text { and } \quad \delta(G) \leq(\gamma-\alpha) n,
$$

then $G$ contains an induced subgraph $H$ satisfying $|H| \geq(1-\beta) n$ and one of the conditions:

(a) $\mu(H)>\gamma(1+\beta \alpha / 2)|H|$;

(b) $\mu(H)>\gamma|H|$ and $\delta(H)>(\gamma-\alpha)|H|$.

Fact 9 ([2], Lemma 6) Let $r \geq 2$ and $G$ be graph a of order $n$. If $G$ contains a $K_{r+1}$ and $\delta(G)>\left(1-1 / r-1 / r^{4}\right) n$, then $j s_{r+1}(G)>n^{r-1} / r^{r+3}$.

Fact 10 ([3], Theorem 2) If $r \geq 2$ and $G$ is a graph of order $n$, then

$$
k_{r}(G) \geq\left(\frac{\mu(G)}{n}-1+\frac{1}{r}\right) \frac{r(r-1)}{r+1}\left(\frac{n}{r}\right)^{r+1} .
$$

Fact 11 ([3], Theorem 4) Let $r \geq 2,0 \leq b \leq 2^{-10} r^{-6}$, and $G$ be a graph of order $n$. If $G$ contains no $K_{r+1}$ and $\mu(G) \geq(1-1 / r-b) n$, then $G$ contains an induced $r$-partite graph $G_{0}$ satisfying $\left|G_{0}\right| \geq\left(1-3 b^{1 / 3}\right) n$ and $\delta\left(G_{0}\right)>\left(1-1 / r-6 b^{1 / 3}\right) n$.

Fact 12 ([12], Theorem 6) Let $r \geq 2,2 / \ln n \leq c \leq r^{-(r+8) r}$, and $G$ be a graph of order $n$. If $G$ contains a $K_{r+1}$ and $\delta(G)>\left(1-1 / r-1 / r^{4}\right) n$, then $G$ contains a $K_{r}^{+}\left(\lfloor c \ln n\rfloor, \ldots,\lfloor c \ln n\rfloor,\left\lceil n^{1-c r^{3}}\right\rceil\right)$.

Fact 13 ([10], Theorem 1) Let $r \geq 2, c^{r} \ln n \geq 1$, and $G$ be a graph of order $n$. If $k_{r}(G) \geq c n^{r}$, then $G$ contains a $K_{r}(s, \ldots s, t)$ with $s=\left\lfloor c^{r} \ln n\right\rfloor$ and $t>n^{1-c^{r-1}}$.

Fact 14 The number of edges of $T_{r}(n)$ satisfies $2 e\left(T_{r}(n)\right) \geq(1-1 / r) n^{2}-r / 4$. 


\section{Proofs}

Below we prove Theorems 1, 2, 4, and 5. We omit the proofs of Theorems 3 and 6 since they are easy consequences of Theorems 2 and 5 .

All proofs have similar simple structure and follow from the facts listed above.

\section{Proof of Theorem 1}

Let $G$ be a graph of order $n$ with $\mu(G)>\mu\left(T_{r}(n)\right)$; thus, by Fact $7, G$ contains a $K_{r+1}$. If

$$
\delta(G)>\left(1-r^{-1}-r^{-4}\right) n,
$$

then, by Fact $9, j s_{r+1}(G)>n^{r-1} / r^{r+3}$, completing the proof.

Thus, we shall assume that (1) fails. Then, letting

$$
\alpha=1 / r^{4}, \quad \beta=1 / 2, \quad \gamma=1-1 / r, \quad K=r / 4,
$$

we see that

$$
\delta(G) \leq(\gamma-\alpha) n
$$

and also, in view of Fact 14,

$$
\mu(G)>\mu\left(T_{r}(n)\right) \geq 2 e\left(T_{r}(n)\right) / n \geq(1-1 / r) n-r / 4 n=\gamma n-K / n .
$$

Given (2), (3) and (4), Fact 8 implies that, for $n \geq r^{15}, G$ contains an induced subgraph $H$ satisfying $|H| \geq n / 2$ and one of the conditions:

(i) $\mu(H)>\left(1-1 / r+1 /\left(4 r^{4}\right)\right)|H|$;

(ii) $\mu(H)>(1-1 / r)|H|$ and $\delta(H)>\left(1-1 / r-1 / r^{4}\right)|H|$.

If condition (i) holds, Fact 10 gives

$$
k_{r+1}(H)>\left(\frac{\mu(H)}{|H|}-1-\frac{1}{r}\right) \frac{r(r-1)}{r+1}\left(\frac{|H|}{r}\right)^{r+1}>\frac{r(r-1)}{4 r^{4}(r+1)}\left(\frac{|H|}{r}\right)^{r+1},
$$

and so,

$$
\begin{aligned}
j s_{r+1}(G) & \geq j s_{r+1}(H) \geq\left(\begin{array}{c}
r+1 \\
2
\end{array}\right) \frac{k_{r+1}(H)}{e(H)}>r(r+1) \frac{k_{r+1}(H)}{|H|^{2}} \\
& >\frac{r(r+1) r(r-1)}{4 r^{4}(r+1) r^{r+1}}|H|^{r-1} \geq \frac{1}{4 r^{r+3}}|H|^{r-1} \geq \frac{1}{2^{r+1} r^{r+3}} n^{r-1} \geq \frac{1}{r^{2 r+4}} n^{r-1},
\end{aligned}
$$

completing the proof.

If condition (ii) holds, then $H$ contains a $K_{r+1}$; thus, $j s_{r+1}(H)>|H|^{r-1} / r^{r+3}$ by Fact 9. To complete the proof, notice that

$$
j s_{r+1}(G)>j s_{r+1}(H)>\frac{|H|^{r-1}}{r^{r+3}} \geq \frac{1}{2^{r-1} r^{r+3}} n^{r-1}>\frac{1}{r^{2 r+4}} n^{r-1} .
$$




\section{Proof of Theorem 2}

Let $G$ be a graph of order $n$ with $\mu(G)>\mu\left(T_{r}(n)\right)$; thus, by Fact $7, G$ contains a $K_{r+1}$. If

$$
\delta(G)>\left(1-1 / r-1 / r^{4}\right) n,
$$

then, by Fact $12, G$ contains a $K_{r}^{+}\left(\lfloor c \ln n\rfloor, \ldots,\lfloor c \ln n\rfloor,\left\lceil n^{1-c r^{3}}\right\rceil\right)$, completing the proof, in view of $c r^{3}<\sqrt{c}$.

Thus, we shall assume that (5) fails. Then, letting

$$
\alpha=1 / r^{4}, \quad \beta=1 / 2, \quad \gamma=1-1 / r, \quad K=r / 4,
$$

we see that

$$
\delta(G) \leq(\gamma-\alpha) n
$$

and also, in view of Fact 14,

$$
\mu(G)>\mu\left(T_{r}(n)\right) \geq 2 e\left(T_{r}(n)\right) / n \geq(1-1 / r) n-r / 4 n=\gamma n-K / n .
$$

Given (6), (7) and (8), Fact 8 implies that, for $n>r^{15}, G$ contains an induced subgraph $H$ satisfying $|H| \geq n / 2$ and one of the conditions:

(i) $\mu(H)>\left(1-1 / r+1 /\left(4 r^{4}\right)\right)|H|$;

(ii) $\mu(H)>(1-1 / r)|H|$ and $\delta(H)>\left(1-1 / r-1 / r^{4}\right)|H|$.

If condition (i) holds, Fact 10 gives

$$
\begin{aligned}
k_{r+1}(H) & >\left(\frac{\mu(H)}{|H|}-1-\frac{1}{r}\right) \frac{r(r-1)}{r+1}\left(\frac{|H|}{r}\right)^{r+1}>\frac{r(r-1)}{4 r^{4}(r+1)}\left(\frac{|H|}{r}\right)^{r+1} \\
& >\frac{1}{2^{r+3} r^{r+4}(r+1)} n^{r+1}>\frac{1}{r^{2 r+9}} n^{r+1} \geq c^{1 /(r+1)} n^{r+1} .
\end{aligned}
$$

Thus, by Fact $13, G$ contains a $K_{r+1}(s, \ldots, s, t)$ with $s=\lfloor c \ln n\rfloor$ and $t>n^{1-c^{r /(r+1)}}>$ $n^{1-\sqrt{c}}$. Then, obviously, $G$ contains a $K_{r}^{+}\left(\lfloor c \ln n\rfloor, \ldots,\lfloor c \ln n\rfloor,\left\lceil n^{1-\sqrt{c}}\right\rceil\right)$, completing the proof.

If condition (ii) holds, then $H$ contains a $K_{r+1}$; thus, by Fact $12, H$ contains a

$$
K_{r}^{+}\left(\lfloor 2 c \ln |H|\rfloor, \ldots,\lfloor 2 c \ln |H|\rfloor,\left\lceil|H|^{1-2 c r^{3}}\right\rceil\right) .
$$

To complete the proof, note that $2 c \ln |H| \geq 2 c \ln \frac{n}{2}>c \ln n$ and

$$
|H|^{1-2 c r^{3}} \geq\left(\frac{n}{2}\right)^{1-2 c r^{3}} \geq \frac{1}{2} n^{1-2 c r^{3}}>n^{1-\sqrt{c}} .
$$

Proof of Theorem 4 Let $G$ be a graph of order $n$ with $\mu(G)>(1-1 / r-b) n$. If $G$ contains no $K_{r+1}$, then condition (b) follows from Fact 11; thus we assume that $G$ contains a $K_{r+1}$. If

$$
\delta(G)>\left(1-1 / r-1 / r^{4}\right) n
$$


then Fact 9 implies condition (a).

Thus, we shall assume that (9) fails. Then, letting

$$
\alpha=1 / r^{4}-b, \quad \beta=4 b / \alpha, \quad \gamma=1-1 / r-b, \quad K=0,
$$

we easily see that

$$
\beta=\frac{4 b}{1 / r^{4}-b} \leq \frac{1}{2}, \quad \delta(G) \leq(\gamma-\alpha) n,
$$

and

$$
\mu(G)>(1-1 / r-b) n=\gamma n .
$$

Given (10), (11) and (12), Theorem 8 implies that, for $n \geq r^{20}, G$ contains an induced subgraph $H$ satisfying $|H| \geq(1-\beta) n$ and one of the conditions:

(i) $\mu(H)>(1-1 / r)|H|$;

(ii) $\mu(H)>(1-1 / r-b)|H|$ and $\delta(H)>\left(1-1 / r-1 / r^{4}\right)|H|$.

If condition (i) holds, by Theorem 1 we have

$$
\begin{aligned}
j s_{r+1}(G) & \geq j s_{r+1}(H) \geq \frac{|H|^{r-1}}{r^{2 r+4}} \geq(1-\beta)^{r-1} \frac{n^{r-1}}{r^{2 r+4}}=\left(1-\frac{4 b}{1 / r^{4}-b}\right)^{r-1} \frac{n^{r-1}}{r^{2 r+4}} \\
& >\left(1-\frac{1}{r^{2}}\right)^{r-1} \frac{n^{r-1}}{r^{2 r+4}} \geq\left(1-\frac{r-1}{r^{2}}\right) \frac{n^{r-1}}{r^{2 r+4}}>\frac{n^{r-1}}{r^{2 r+5}},
\end{aligned}
$$

implying condition (a) and completing the proof.

Suppose now that condition (ii) holds. If $H$ contains a $K_{r+1}$, by Fact 9 , we see that

$$
j s_{r+1}(G) \geq j s_{r+1}(H) \geq \frac{|H|^{r-1}}{r^{r+3}} \geq(1-\beta)^{r-1} \frac{n^{r-1}}{r^{r+3}}>\frac{n^{r-1}}{2^{r-1} r^{r+3}}>\frac{n^{r-1}}{r^{2 r+5}},
$$

implying condition (a).

If $H$ contains no $K_{r+1}$, by Fact $11, H$ contains an induced $r$-partite subgraph $H_{0}$ satisfying $\left|H_{0}\right|>\left(1-3 b^{1 / 3}\right)|H|$ and $\delta\left(H_{0}\right)>\left(1-6 b^{1 / 3}\right)|H|$. Now from

$$
\beta=\frac{4 b}{1 / r^{4}-b} \leq \frac{4 b}{1 / r^{4}-1 /\left(2^{10} r^{6}\right)} \leq 8 r^{4} b<b^{1 / 3},
$$

we deduce that

$$
\left|H_{0}\right| \geq\left(1-3 b^{1 / 3}\right)|H| \geq\left(1-3 b^{1 / 3}\right)(1-\beta) n>\left(1-4 b^{1 / 3}\right) n
$$

and

$$
\delta\left(H_{0}\right) \geq\left(1-6 b^{1 / 3}\right)|H| \geq\left(1-6 b^{1 / 3}\right)(1-\beta) n>\left(1-7 b^{1 / 3}\right) n .
$$

Thus condition (b) holds, completing the proof.

Proof of Theorem 5 Let $G$ be a graph of order $n$ with $\mu(G)>(1-1 / r-b) n$. If $G$ contains no $K_{r+1}$, then condition (b) follows from Fact 11; thus we assume that $G$ contains a $K_{r+1}$. If

$$
\delta(G)>\left(1-1 / r-1 / r^{4}\right) n,
$$


then Fact 12 implies condition (a).

Thus, we shall assume that (13) fails. Then, letting

$$
\alpha=1 / r^{4}-b, \quad \beta=4 b / \alpha, \quad \gamma=1-1 / r-b, \quad K=0,
$$

we easily see that

$$
\beta=\frac{4 b}{1 / r^{4}-b} \leq \frac{1}{2}, \quad \delta(G) \leq(\gamma-\alpha) n,
$$

and

$$
\mu(G)>(1-1 / r-b) n=\gamma n .
$$

Given (14), (15) and (16), Theorem 8 implies that, for $n \geq r^{20}, G$ contains an induced subgraph $H$ satisfying $|H| \geq(1-\beta) n$ and one of the conditions:

(i) $\mu(H)>(1-1 / r)|H|$;

(ii) $\mu(H)>(1-1 / r-b)|H|$ and $\delta(H)>\left(1-1 / r-1 / r^{4}\right)|H|$.

If condition (i) holds, Theorem 2 implies that $H$ contains a

$$
K_{r}^{+}\left(\lfloor 2 c \ln |H|\rfloor, \ldots,\lfloor 2 c \ln |H|\rfloor,\left[|H|^{1-2 c r^{3}}\right\rceil\right) .
$$

Now condition (a) follows in view of $2 c \ln |H| \geq 2 c \ln \frac{n}{2}>c \ln n$ and

$$
|H|^{1-2 c r^{3}} \geq\left(\frac{n}{2}\right)^{1-2 c r^{3}} \geq \frac{1}{2} n^{1-2 c r^{3}}>n^{1-\sqrt{c}},
$$

completing the proof.

Suppose now that condition (ii) holds. If $H$ contains a $K_{r+1}$, by Fact $12, H$ contains a

$$
K_{r}^{+}\left(\lfloor 2 c \ln |H|\rfloor, \ldots,\lfloor 2 c \ln |H|\rfloor,\left[|H|^{1-2 c r^{3}}\right\rceil\right) .
$$

This implies condition (a) in view of $2 c \ln |H| \geq 2 c \ln \frac{n}{2}>c \ln n$ and

$$
|H|^{1-2 c r^{3}} \geq\left(\frac{n}{2}\right)^{1-2 c r^{3}} \geq \frac{1}{2} n^{1-2 c r^{3}}>n^{1-\sqrt{c}} .
$$

If $H$ contains no $K_{r+1}$, the proof is completed as the proof of Theorem 4.

Acknowledgement. Thanks are due to the referee for careful reading and useful remarks.

\section{References}

[1] B. Bollobás, Modern Graph Theory, Graduate Texts in Mathematics, 184, SpringerVerlag, New York (1998).

[2] B. Bollobás, V. Nikiforov, Joints in graphs, to appear in Discrete Math. 
[3] B. Bollobás, V. Nikiforov, Cliques and the Spectral Radius, J. Combin. Theory Ser. B. 97 (2007), 859-865.

[4] P. Erdős, On the structure of linear graphs, Israel J. Math. 1 (1963), 156-160.

[5] P. Erdős, Some recent results on extremal problems in graph theory (results) in Theory of Graphs (Internat. Sympos., Rome, 1966), pp. 117-130, Gordon and Breach, New York; Dunod, Paris.

[6] P. Erdős, On some new inequalities concerning extremal properties of graphs, in: Theory of Graphs (Proc. Colloq., Tihany, 1966), pp. 77-81, Academic Press, New York, 1968.

[7] P. Erdös, On the number of complete subgraphs and circuits contained in graphs, Časopis Pěst. Mat. 94 (1969), 290-296.

[8] P. Erdős, R.J. Faudree, C.C. Rousseau, Extremal problems and generalized degrees, Discrete Math. 127 (1994), 139-152.

[9] P. Erdős, M. Simonovits, On a valence problem in extremal graph theory, Discrete Math. 5 (1973), p. 323-334.

[10] V. Nikiforov, Graphs with many $r$-cliques have large complete $r$-partite subgraphs, Bull. of London Math. Soc. 40 (2008), 23-25. Update available at http://arxiv.org/math.CO/0703554

[11] V. Nikiforov, Stability for large forbidden graphs, to appear in J. Graph Theory. Preprint available at http://arxiv.org/abs/0707.2563

[12] V. Nikiforov, Turán's theorem inverted, submitted for publication. Preprint available at http://arxiv.org/abs/0707.3439

[13] V. Nikiforov, Some inequalities for the largest eigenvalue of a graph, Combin. Probab. Comp. 11 (2002), 179-189.

[14] V. Nikiforov, The smallest eigenvalue of $K_{r}$-free graphs, Discrete Math. 306 (2006), 612-616.

[15] V. Nikiforov, Bounds on graph eigenvalues II, Linear Algebra Appl. 427 (2007) 183189.

[16] V. Nikiforov, A spectral condition for odd cycles, Linear Algebra Appl. 428 (2008), 1492-1498. Update available at http://arxiv.org/abs/0707.4499

[17] V. Nikiforov, More spectral bounds on the clique and independence numbers, to appear in J. Combin. Theory Ser. B. Preprint available at http://arxiv.org/abs/0706.0548

[18] V. Nikiforov, A spectral Erdős-Stone-Bollobás theorem, to appear in Combin. Probab. Comput. Preprint available at http://arxiv.org/abs/0707.2259

[19] M. Simonovits, A method for solving extremal problems in graph theory, stability problems, in: Theory of Graphs (Proc. Colloq., Tihany, 1966), pp. 279-319, Academic Press, New York, 1968. 